Präv Gesundheitsf 2012 · 7:227-228

DOI 10.1007/s11553-012-0362-1

Online publiziert: 27. Oktober 2012

(c) Springer-Verlag Berlin Heidelberg 2012

\section{Michael Rädel}

Poliklinik für Zahnärztliche Prothetik, Medizinische Fakultät Carl Gustav Carus,

Technische Universität Dresden, Dresden, Deutschland

\section{Editorial}

Sehr geehrte Leserinnen und Leser,

vor einigen Tagen fiel mir eine Ausgabe der US-amerikanischen Tageszeitung USA-Today in die Hände. Darin befragten Reporter dieser Zeitung namhafte Wissenschaftler verschiedenster Fachbereiche über ihre Vorstellung vom menschlichen Leben in der Zukunft. Dabei las ich mehr beiläufig als interessiert über neue Wohn-, Mobilitäts- und Kommunikationskonzepte. Beim Stichpunkt Medizin und Gesundheit hielt ich inne, denn dort hieß es: Die Medizin wird wahrscheinlich zum Großteil präventiv ausgerichtet sein. Die befragte Expertin war sich sicher, dass die meisten heute bekannten Krankheiten in 30 und mehr Jahren bereits vor der Erkrankung verhindert würden. Auch wenn diese These ein wenig euphorisch wirkt, kann eine immer weiter wachsende Bedeutung der Prävention in der Medizin wohl kaum geleugnet werden. Dabei gilt es aber auch, maßgeschneiderte Präventionskonzepte - nicht nur für verschiedene Erkrankungen - sondern auch für verschiedene Gruppen innerhalb der Bevölkerung zu entwickeln.

Allein die aufeinanderfolgenden Lebens- und Entwicklungsphasen des Menschen, welche unterschiedlicher nicht sein könnten, verlangen nach individuellen Konzepten, damit präventive Maßnahmen ihre Wirksamkeit entfalten können. In diesem Sinne sind für Sie unter dem großen Titel „Prävention und Gesundheitsforschung" in diesem Heft wieder eine große Bandbreite interessanter Beiträge zusammengefasst, welche verschiedenste Altersgruppen - vom Kindergartenkind bis zum Senioren - umfassen.
Dass wenige gezielte Schulungen von Kindergartenkindern in wertschätzender Kommunikation einen positiven Einfluss auf das Verhalten der Untersuchten haben können, zeigt Herr Schoppe aus Leipzig. Aus dem Hochschulbereich wird im Beitrag von Dr. Gusy berichtet: Er geht der interessanten Fragestellung nach, ob Bachelor-/Masterstudierende stärker „ausgebrannt" sind, als Studierende der klassischen Studiengänge. Innerhalb der andauernden Diskussion über die Umsetzung des Bologna-Prozesses sind derartige Betrachtungen sicherlich sehr sinnvoll. Die Zielgruppe „Kinder und Jugendliche“ ist im Fokus des Beitrags von Keydel, welcher bundesweite Handlungsprogramme für diese Altersgruppe kritisch betrachtet und analysiert. Kinder, Jugendliche und Studenten stellen sich aber nicht nur schulischen oder universitären Herausforderungen. Auch sportliche Betätigung ist für die meisten ein unbedingter Bestandteil des täglichen Lebens. Der Effekt eines Sportmundschutzes beim Boxen auf die Wirbelsäulenstellung wird daher im Beitrag von Frau Dr. Ohlendorf aufgegriffen. Die Zielgruppe „Familie“ wird durch Frau Dr. Kunz thematisiert. Sie berichtet von einer Untersuchung in Risikofamilien mit depressiver Symptomatik der Eltern. Die Stressbelastung der Eltern spielt darin eine zentrale Rolle, was die Bedeutung des Beitrags in Zeiten zunehmender Stress- und Burnout-Phänomene unterstreicht. Die provokante Frage „Kann Gesundheit Sünde sein?" erregt sicherlich unser aller Interesse. Herr Dr. Fallmann aus Klagenfurt versucht in seiner Analyse dabei eine Antwort zu finden, warum die meisten Menschen nicht gesund leben - es aber trotzdem tun sollten. Viel- leicht ergeben sich ja in Zukunft Möglichkeiten, dem Menschen das "Vernünftige“ und "Gesunde" ebenso reizvoll und interessant zu machen, wie es das „Verbotene“ und „Ungesunde“ schon ewig ist? Ein faszinierender Gedanke!

Aber auch der Zielgruppe der Senioren wird in diesem Heft Aufmerksamkeit geschenkt. Herr Teti aus Berlin berichtet von einer Befragung zur individuellen Wohnmobilität bei älteren Menschen und analysiert dabei Sesshaftigkeit und Umzugsbereitschaft.

Ärztliche Diagnosen mit psychischem oder psychosomatischem Hintergrund sind in den vergangenen Jahren stark gestiegen. Demzufolge nimmt auch die Zahl der dadurch begründeten Arbeitsunfähigkeit von Arbeitnehmern zu. Aus diesem aktuellen Grund hat Frau Pohrt den Zusammenhang zwischen Arbeitsunfähigkeit und psychischen Faktoren wie Arbeitsplatzzufriedenheit und Arbeitsunsicherheit untersucht. Mit der Kommunikation im betrieblichen Gesundheitsmanagement beschäftigt sich der anschlieBende Beitrag von Walter et al. Einen interessanten Kommentar zu Auswirkungen des Menschenhandels auf die betroffenen Frauen hat Frau Zühlke verfasst. Sie analysiert sehr gewissenhaft die Situation, zeigt aber auch Handlungsoptionen für konfrontierte Ärzte auf. Ein zusammenfassender Beitrag von Prof. Hartmann rundet das Heft ab und stellt die verschiedenen Gesundheitsziele in den deutschen Bundesländern dar. Er skizziert diese, analysiert daraus resultierende Spannungen und eröffnet mögliche Verbesserungen durch bessere Abstimmung - sicherlich ein nicht zu unterschätzendes Potenzial. 


\section{Editorial}

Ich wünsche Ihnen, verehrte Leserinnen und Leser, zahlreiche neue Erkenntnisse, den einen oder anderen Gedankenansatz und natürlich eine entspannte Lesezeit für die Lektüre dieses Heftes. Gönnen Sie sich ein paar ruhige Minuten für die interessanten Beiträge - ganz im Sinne der Prävention vor Stress- oder BurnoutPhänomenen. Denn: Dieses Heft zu lesen, kann mit Sicherheit nicht Sünde sein! Mit herzlichen Grüßen aus Dresden

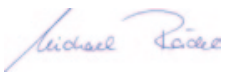

\section{Korrespondenzadresse}

Dr. med. dent. M. Rädel, Msc.
Poliklinik für Zahnärztliche
Prothetik
Medizinische Fakultät
Carl Gustav Carus
Technische Universität
Dresden
Fetscherstraße 74,
01307 Dresden
Michael.Raedel@uniklinikum-
dresden.de

